NASA-CR-204686

\title{
Self-consistent superthermal electron effects on plasmaspheric refilling
}

\author{
M. W. Liemohn' \\ Space Physics Research Laboratory, University of Michigan, Ann Arbor \\ G. V. Khazanov and T. E. Moore \\ Space Sciences Laboratory, NASA Marshall Space Flight Center, Huntsville, Alabama \\ S. M. Guiter \\ Center for Space Plasma and Aeronomic Research, University of Alabama in Huntsville
}

\begin{abstract}
The effects of self-consistently including superthermal electrons in the definition of the ambipolar electric field are investigated for the case of plasmaspheric refilling after a geomagnetic storm. By using the total electron population in the hydrodynamic equations, a method for incorporating superthermal electron parameters in the electric field and electron temperature calculation is developed. Also, the ambipolar electric field is included in the kinetic equation for the superthermal electrons through a change of variables using the total energy and the first adiabatic invariant. Calculations based on these changes are performed by coupling time-dependent models of the thermal plasma and superthermal electrons. Results from this treatment of the electric field and the self-consistent development of the solution are discussed in detail. Specifically, there is a decreased thermal electron density in the plasmasphere during the first few minutes of refilling, a slightly accelerated proton shock front, and a decreased superthermal electron flux due to the deceleration by the electric field. The timescales of plasmaspheric refilling are discussed and determined to be somewhat shorter than previously calculated for the thermal plasma and superthermal electron population due to the effects of the field-aligned potential.
\end{abstract}

\section{Introduction}

The plasmasphere is the region of near-Earth space where geomagnetic flux tubes corotate with the Earth [Nishida, 1966]. The convection electric field sweeps away the flux tubes outside the plasmasphere, never allowing them to fill to a steady state level of thermal plasma. During a geomagnetic disturbance, the plasmasphere is reduced due to the increased convection, sweeping away the plasma in the flux tubes that were recently corotating. When the convection decreases, these flux tubes return to their corotating trajectories around the Earth, and refilling of the thermal plasma can begin (see reviews by Horwitz [1987]; Singh and Horwitz [1992]; and Singh et al. [1994]).

During refilling after a geomagnetic storm, the ions can flow from the ionosphere into the plasmasphere at supersonic speeds [Banks et al., 1971; Khazanov et al., 1984; Singh et al., 1986; Rasmussen and Schunk, 1988; Guiter and Gombosi, 1990; Gorbachev et al., 1991; Wilson et al., 1992; Guiter et al., 1995], resulting in shock formations. The flow conditions vary dramatically along a flux tube, from a subsonic, $\mathrm{O}^{+}$

\footnotetext{
${ }^{1}$ Now at Space Sciences Laboratory, NASA Marshall Space Flight Center, Huntsville.
}

Copyright 1997 by the American Geophysical Union.

Paper number $96 \mathrm{JA03962.}$

0148-0227/97/96JA-03962\$09.00 dominated, collisional region at low altitudes to a supersonic, $\mathrm{H}^{+}$dominated, collisionless region at high altitudes. In the high-altitude region, highly non-Maxwellian distributions develop as the particles stream into the plasmasphere. The high mobility of the electrons allows them to outrun the ions; this violates charge neutrality, however, and an electric field appears to force the electrons to move with the ions (see Figure 1). This drag on the electrons is also an acceleration mechanism for the ions, which changes the potential structure.

Superthermal electrons are created in the ionosphere by photoionization and impact ionization of atmospheric neutrals. These electrons, which have a highly structured source function and represent a nonthermal tail in the electron distribution function, must be treated kinetically in plasma models [Khazanov et al., 1994]. They are affected by the electric field and the accelerated ions and can redistribute the potential along the field line, and a self-consistent calculation should be performed in order to model the dynamics of this phenomenon correctly.

A recent approach to coupling the thermal plasma populations is to calculate the electron density and velocity from the conditions of quasi-neutrality and current balance, and then find the parallel electric field and the electron temperature from the electron momentum and energy equations. These values are then used in the equations for the other species. This type of calculation was performed for the inner magnetosphere [Khazanov et al., 1984; Richards and Torr, 1986; Guiter and Gombosi, 1990; Gorbachev et al., 1991; Miller et al., 1993; 


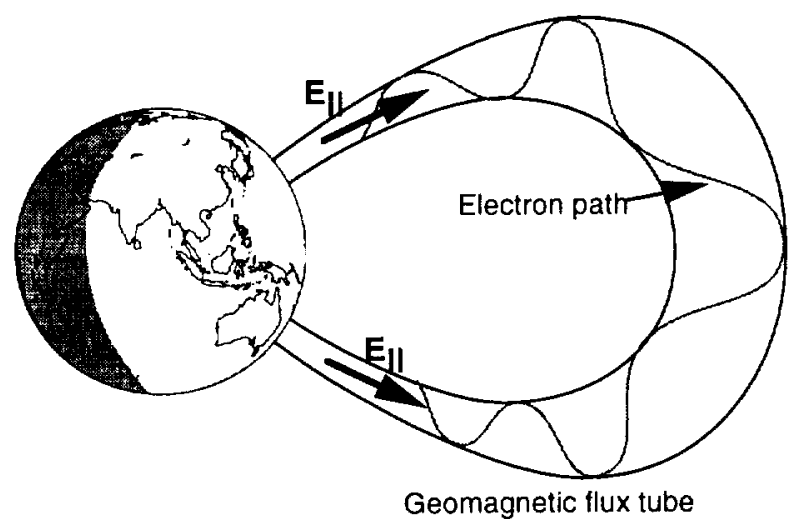

Figure 1. Schematic of a plasmaspheric flux tube, showing the helical path of a superthermal electron and the usual direction of the ambipolar electric field.

Singh et al., 1994; Guiter et al. 1995], the topside ionosphere in the auroral region [Min et al., 1993], and the polar ionosphere [Schunk and Watkins, 1981; Gombosi and Nagy, 1989; Tam et al., 1995].

Several of these models calculate superthermal electrons and the thermal plasma simultaneously at various levels of selfconsistency. The model of Khazanov et al. [1984] calculates the thermal plasma with a time-dependent 16-moment transport model for the electrons and $\mathrm{O}^{+}$and $\mathrm{H}^{+}$ions, along with a steady state, superthermal electron kinetic model for thermal heating rates. The field line interhemispheric plasma (FLIP) model [Richards and Torr, 1986] calculates the thermal plasma with a time-dependent five-moment fluid model and uses a steady state two-stream model for the superthermal electrons [e.g., Nagy and Banks, 1970] for the heating rates. The model of Gorbachev et al. [1991] includes wave activity in its 13moment equation set for the thermal plasma calculation, obtaining slightly different results, especially in the morning/evening sectors when refilling/depletion of the flux tube occurs. Min et al. [1993] calculate $E_{||}$from the thermal plasma equations and then use this electric field in the steady state kinetic equation for the superthermal electrons in the aurora and at midlatitudes. Most recently, Tam et al. [1995] treat the ions and superthermal electrons kinetically along with a fluid approach for the thermal electrons, and obtain steady state polar wind results that are collisionally and electrodynamically self-consistent.

The goal of this study is to extend results such as these to a time-dependent calculation while collisionally and electrodynamically coupling the plasma populations. This will involve a reexamination of the coupling processes between the superthermal electrons and the thermal plasma, resulting in various terms linking the equation sets. A driver program is used that can couple any thermal plasma model to the superthermal electron model of Khazanov and Liemohn [1995]. For this study, the two-stream, field-aligned, fivemoment hydrodynamic model of Guiter et al. [1995] will be used for the thermal plasma calculation. Since plasmaspheric refilling along closed field lines is the focus of this study, this model was chosen for its ability to calculate the interpenetrating streams of ions from the conjugate hemispheres of the flux tube.

We realize that there is some discussion as to whether a hydrodynamic treatment is valid for plasmaspheric refilling. For instance, Schulz and Koons [1972] argued that collision frequencies are not nearly high enough to justify a fluid treatment of even the thermal ions, recommending a kinetic approach to modeling plasmaspheric refilling. Performing a completely kinetic calculation for the superthermal and thermal plasma populations is beyond the scope of the present study, however, and we must accept the limitations of hydrodynamic modeling for the thermal plasma until a more comprehensive kinetic model is developed. It should be noted, however, that Singh et al. [1994] found similar results between a hydrodynamic and semikinetic model of plasmaspheric refilling for the first hour, and so we will discuss a similar timeframe.

We will discuss the inclusion of the total electron component in the fluid equations (section 2), followed by a description of the coupling processes in the superthermal electron model (sections 3 and 4). Results are presented for the case of plasmaspheric refilling along an $L=4$ flux tube after a density depletion (section 5), closing with a discussion of the results, their implications, and further use (section 6).

\section{Total Electron Component in the Hydrodynamic Equations}

The hydrodynamic thermal plasma model is described by Guiter et al. [1995]. It is a five-moment fluid description for the $\mathrm{H}^{+}$and $\mathrm{O}^{+}$ions, treating the ions created in the conjugate hemispheres as distinct populations (two-stream thermal model). The electron density is calculated by assuming quasineutrality, the electron bulk speed by imposing a zero current condition, and the electron temperature from the energy equation. These equations are solved along a geomagnetic field line under nonsteady conditions. Important modifications have been made to this model, however, which will now be discussed.

The basic approach to the thermal electron equations used here is the same as before, using the four equations of quasineutrality, zero current, electron momentum, and electron energy to find the four variables $n_{e}, u_{e}, E_{\|}$, and $T_{e}$. However, the form of these equations used by Guiter et al. [1995] assumes that the only electron species is the thermal electron population. In a calculation where the electrons are split into thermal and superthermal populations, both of these components must be taken into account in all four of these equations. In a relatively dense plasma of a filled flux tube, the superthermal contribution will be small, but during transient events where the thermal plasma population is depleted, the superthermal electrons could significantly affect the thermal plasma parameters.

By including superthermal electrons, the condition of quasineutrality now has the form

$$
n_{e}+n_{s}=\sum_{i} n_{i}
$$

and the zero current assumption becomes

$$
n_{e} u_{e}+n_{s} u_{s}=\sum_{i} n_{i} u_{i}
$$

where $n_{s}$ and $n_{s} u_{s}$ are the superthermal electron density and flux, respectively, found by taking the appropriate velocityspace integral of the superthermal electron flux, $\phi$. Examination of (1) and (2) shows that the presence of superthermal electron terms will decrease the corresponding thermal electron parameters. 
In deriving the electron moment equations, the electron distribution function, $f$, can be separated into two parts: the thermal electrons, $f_{e}$; and the superthermal electrons, $f_{s}$ (for a complete discussion of this, see Khazanov [1979]). The momentum and energy equations then become

$$
\begin{aligned}
& m_{e} \frac{\partial}{\partial t}\left(n_{e} u_{e}+n_{s} u_{s}\right)+m_{e} B \frac{\partial}{\partial s}\left[\frac{1}{B}\left(n_{e} u_{e}^{2}+n_{s} u_{s}^{2}\right)\right] \\
& +\frac{\partial}{\partial s}\left(P_{e}+P_{s}\right)=m_{e}\left(n_{e}+n_{s}\right)\left(\frac{q_{e}}{m_{e}} E_{\mid 1}+g_{11}\right) \\
& +\frac{\delta M_{e}}{\delta t}+\frac{\delta M_{s}}{\delta t}+u_{s} S_{s} \\
& \frac{\partial}{\partial t}\left(\frac{m_{e} n_{e} u_{e}^{2}}{2}+\frac{3 P_{e}}{2}+\frac{m_{e} n_{s} u_{s}^{2}}{2}+\frac{3 P_{s}}{2}\right) \\
& +B \frac{\partial}{\partial s}\left[\frac{1}{B}\left(\frac{m_{e} n_{e} u_{e}^{3}}{2}+\frac{5 u_{e} P_{e}}{2}+Q_{e}+\frac{m_{e} n_{s} u_{s}^{3}}{2}+\frac{5 u_{s} P_{s}}{2}+Q_{s}\right)\right](4) \\
& =m_{e}\left(n_{e} u_{e}+n_{s} u_{s}\right)\left(\frac{q_{e}}{m_{e}} E_{\mid 1}+g_{\| 1}\right)+\frac{\delta E_{e}}{\delta t}+u_{e} \frac{\delta M_{e}}{\delta t}+\frac{\delta E_{s}}{\delta t} \\
& +u_{s} \frac{\delta M_{s}}{\delta t}+\frac{u_{s}^{2}}{2} S_{s}
\end{aligned}
$$

where $B$ is the geomagnetic field strength; $P_{\alpha}$ and $Q_{\alpha}$ are the pressure and heat flux, respectively, of component $\alpha$; and the collision terms $\left(\delta M_{e} / \delta t, \delta M_{s} / \delta t, \delta E_{e} / \delta t\right.$, and $\left.\delta E_{s} / \delta t\right)$ represent interactions between the electrons and all other species (ions and neutral particles). The superthermal electron source $S_{s}$ is included in the kinetic equation as a differential source term which includes primary, secondary, and tertiary superthermal electron production calculated from an EUV solar spectrum and photoabsorption and impact ionization cross sections of the atmospheric species [Khazanov and Liemohn, 1995]. For conservation of mass, the local production of superthermal electrons must balance the local production of ions,

$$
S_{s}=\sum_{i} S_{i}
$$

where $S_{i}$ contributes to the ion moment equations.

The superthermal electron distribution function, however, is calculated by numerically solving the kinetic equation (this will be discussed later). We can therefore find the momentum and energy conservation for the superthermal electron component,

$$
\begin{aligned}
& m_{e} \frac{\partial\left(n_{s} u_{s}\right)}{\partial t}+m_{e} B \frac{\partial}{\partial s}\left(\frac{n_{s} u_{s}^{2}}{B}\right)+\frac{\partial P_{s}}{\partial s} \\
& =m_{e} n_{s}\left(\frac{q_{e}}{m_{e}} E_{11}+g_{11}\right)+\frac{\delta M_{s}}{\delta t}+u_{s} S_{s}+\left(\frac{\delta M_{e s}}{\delta t}\right)_{C C}-u_{e} S_{e} \\
& \frac{\partial}{\partial t}\left(\frac{m_{e} n_{s} u_{s}^{2}}{2}+\frac{3 P_{s}}{2}\right)+B \frac{\partial}{\partial s}\left[\frac{1}{B}\left(\frac{m_{e} n_{s} u_{s}^{3}}{2}+\frac{5 u_{s} P_{s}}{2}+Q_{s}\right)\right] \\
& =m_{e} n_{s} u_{s}\left(\frac{q_{e}}{m_{e}} E_{11}+g_{11}\right)+\frac{\delta E_{s}}{\delta t}+u_{s} \frac{\delta M_{s}}{\delta t}+\frac{u_{s}^{2}}{2} S_{s} \\
& \quad+\left(\frac{\delta E_{e s}}{\delta t}\right)_{C C}-\frac{u_{e}^{2}}{2} S_{e}
\end{aligned}
$$

where the "es" collision terms represent the momentum and energy transfer terms due to Coulomb collisions from the thermal electrons to the superthermal electrons. The final term in these two equations represent superthermal electron losses as particles cascade down to low energies and become part of the thermal electron population. Subtracting these equations from (3) and (4) yields thermal electron equations similar to those of Guiter and Gombosi [1990],

$$
\begin{aligned}
& m_{e} \frac{\partial\left(n_{e} u_{e}\right)}{\partial t}+m_{e} B \frac{\partial}{\partial s}\left(\frac{n_{e} u_{e}^{2}}{B}\right)+\frac{\partial P_{e}}{\partial s} \\
& =m_{e} n_{e}\left(\frac{q_{e}}{m_{e}} E_{\|}+g_{\| 1}\right)+\frac{\delta M_{e}}{\delta t}+u_{e} S_{e}+\left(\frac{\delta M_{s e}}{\delta t}\right)_{C C} \\
& \frac{\partial}{\partial t}\left(\frac{m_{e} n_{e} u_{e}^{2}}{2}+\frac{3 P_{e}}{2}\right)+B \frac{\partial}{\partial s}\left[\frac{1}{B}\left(\frac{m_{e} n_{e} u_{e}^{3}}{2}+\frac{5 u_{e} P_{e}}{2}+Q_{e}\right)\right] \\
& =m_{e} n_{e} u_{e}\left(\frac{q_{e}}{m_{e}} E_{\mid I}+g \|\right)+\frac{\delta E_{e}}{\delta t}+u_{e} \frac{\delta M_{e}}{\delta t} \\
& +\frac{u_{e}^{2}}{2} S_{e}+\left(\frac{\delta E_{s e}}{\delta t}\right)_{C C}
\end{aligned}
$$

where the last terms in (5) and (6) account for momentum and energy transfer from the superthermal electrons to the thermal electrons through Coulomb interactions, and have the form

$$
\begin{gathered}
\left(\frac{\delta M_{s e}}{\delta t}\right)_{C C}=-\left(\frac{\delta M_{e s}}{\delta t}\right)_{C C}=-\int m v_{11} S_{e e} d E d \Omega \\
=2 \beta A n_{e}\left[\frac{\left(\Delta \phi_{11}\right)_{E_{\min }}}{\sqrt{E_{\min }}}\left(1-\frac{T_{e}}{2 E_{\min }}\right)+\int_{E_{\min }}^{\infty} \frac{\Delta \phi_{11}}{E^{3 / 2}}\left(1+\frac{T_{e}}{4 E}\right) d E\right] \\
\left(\frac{\delta E_{s e}}{\delta t}\right)_{C C}=-\left(\frac{\delta E_{e s}}{\delta t}\right)_{C C}=-\int E S_{e e} d E d \Omega \\
=4 \pi A n_{e}\left[\phi_{E_{\min }}\left(1-\frac{2 T_{e}}{E_{\min }}\right)+\int_{E_{\min }}^{\infty} \frac{\phi}{E} d E\right]
\end{gathered}
$$

where $E$ is energy; $\phi$ is the superthermal electron flux, $\phi=2 E f / m^{2} ; \beta=1.7 \times 10^{-8} \mathrm{eV}^{1 / 2} \mathrm{~s} \mathrm{~cm}^{-1} ; A=2 \pi \mathrm{e}^{4} \ln \Lambda=2.6 \times 10^{-12}$ $\mathrm{eV}^{2} \mathrm{~cm}^{2}$; and $\Delta \phi_{\|}$is the net directional flux along the field line,

$$
\Delta \phi_{||}=2 \pi \int_{-1}^{1} \mu \phi d \mu
$$

where $\mu$ is the cosine of the pitch angle, defined as the angle between the geomagnetic field and the particle velocity. The energy $E_{\min }$ is the lowest energy of the superthermal electron distribution where the thermal and superthermal distribution functions intersect. (Note that $E_{||}$is the field-aligned electric field, whereas $E$ or $E_{\alpha}$ is kinetic energy.) Equation (8) is the definition of the thermal electron heating rate due to Coulomb collisions with superthermal electrons.

Using (2) and the thermal ion momentum equations, the electron momentum equation (5) can be rewritten and solved for $E_{l \mid}$, 


$$
\begin{aligned}
E_{11}= & -\frac{1}{e n_{e}}\left[\frac{\partial}{\partial s}\left(p_{e}+\rho_{e} u_{e}^{2}-\sum_{i} \frac{m_{e}}{m_{i}}\left(p_{i}+\rho_{i} u_{i}^{2}\right)\right)-\frac{\partial\left(\rho_{s} u_{s}\right)}{\partial t}\right. \\
& -\frac{\delta M_{e}}{\delta t}-\left(\frac{\delta M_{s e}}{\delta t}\right)_{C C}+\sum_{i} \frac{m_{e}}{m_{i}}\left(\frac{\delta M_{i}}{\delta t}-\left(u_{e}-u_{i}\right) S_{i}\right) \\
& \left.-\frac{1}{B} \frac{\partial B}{\partial s}\left(\rho_{e} u_{e}^{2}-\sum_{i} \frac{m_{e}}{m_{i}} \rho_{i} u_{i}^{2}\right)\right]
\end{aligned}
$$

where $\rho_{e}=m_{e} n_{e}, p_{e}=n_{e} k_{B} T_{e}$, and $T_{e}$ is found from the electron energy equation (6), rewritten as a diffusion equation,

$$
\begin{aligned}
\rho_{e} \frac{\partial T_{e}}{\partial t} & =\frac{2}{3} \frac{m_{e}}{A k_{B}} \frac{\partial}{\partial s}\left(A \kappa_{e} \frac{\partial T_{e}}{\partial s}\right)-T_{e}\left[S_{e}+\frac{2}{3} \frac{\rho_{e}}{A}+\frac{\partial\left(A u_{e}\right)}{\partial s}\right] \\
& -\rho_{e} u_{e} \frac{\partial T_{e}}{\partial s}+\frac{2}{3} \frac{m_{e}}{k_{B}}\left[\frac{\delta E_{e}}{\delta t}+\left(\frac{\delta E_{s e}}{\delta t}\right)_{C C}\right]
\end{aligned}
$$

where $Q_{e}$ has been replaced by the thermal conductivity flux according to Banks and Kockarts [1973]. Equation (9) is equivalent to the multiple-electron-species electric field derived in Mitchell et al. [1990]. Equations (1) and (2) can be used to find the thermal electron density and velocity in (9) and (10), and these four equations provide the basis for the coupling of the two components.

\section{Superthermal Electron Kinetic Equation in the Presence of an Electric Field}

A detailed description of the superthermal electron transport model is given by Khazanov et al. [1993, 1994] and Khazanov and Liemohn [1995], numerically solving the time-dependent, guiding center kinetic equation along a geomagnetic field line. These studies, however, omitted electric fields. The guiding center kinetic equation in the presence of a magnetic fieldaligned electric field is [cf. Khazanov et al., 1994, Equation (2)]

$$
\begin{aligned}
& \frac{\beta}{\sqrt{E}} \frac{\partial \phi}{\partial t}+\mu \frac{\partial \phi}{\partial s}-\frac{1-\mu^{2}}{2}\left(-\frac{F}{E}+\frac{1}{B} \frac{\partial B}{\partial s}\right) \frac{\partial \phi}{\partial \mu} \\
& +E F \mu \frac{\partial}{\partial E}\left(\frac{\phi}{E}\right)=q+\langle S\rangle
\end{aligned}
$$

where $F$ represents field-aligned forces such as that from an electric field, $F=-e E_{\|} ; s$ is distance along the magnetic field; $q$ is the source term; and $\langle S\rangle$ represents the collision operators [e.g., Khazanov and Liemohn, 1995, Equations (2)-(6)].

The electric field alters the magnitude of the parallel velocity for a given particle. As can be seen from (11), inclusion of $E_{\text {II }}$ introduces a drift of the particles in velocity space (drifts in energy and pitch angle) as it changes $v_{\| l}$. For instance, if $E_{\|}$is directed upward, then upward flowing electrons will be decelerated, shifting to larger pitch angles and lower energies, while the downward flowing electrons will be accelerated, shifting to smaller pitch angles and higher energies, as shown in Figure 2. Since both $E$ and $\mu$ depend on $v_{\|}$, the magnitude of the shift in terms of energy and pitch angle is different for each point in $E-\mu$ space. Field-aligned particles will have very little shift in pitch angle, while particles with $v_{\perp} \gg v_{\|}$will have very little change in energy. In Figure 2, an electron starting with energy $E_{3}$ and pitch angle $\theta_{1}$ will move to energy $E_{2}$ and pitch angle $\theta_{2}$, while an electron starting at $E_{2}$ and $\theta_{1}$ will end up at

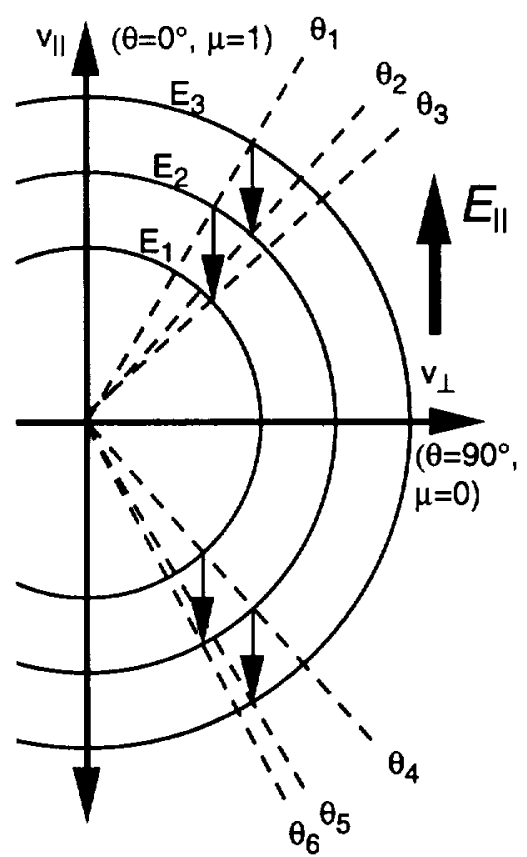

Figure 2. Schematic of the drift in velocity space due to a magnetic field-aligned electric field.

$E_{l}$ and $\theta_{3}$. The energy difference between $E_{1}$ and $E_{2}$ is similar to that between $E_{2}$ and $E_{3}$, since both particles started at the same pitch angle and the energy dependence is weak, but the resulting pitch angles are different because this drift depends on both energy and pitch angle. A similar diagram is shown for downward flowing electrons.

Along a geomagnetic field line, the ambipolar electric field generally points from each ionosphere toward the equatorial plane as plasma is produced in the ionosphere and moves upward (Figure 1). Thus electrons are typically decelerated for the first half of their plasmaspheric journey, and then accelerated by roughly the same amount through the second half of the plasmasphere. The magnetic field $B$ however, also acts upon the particles, focusing them in pitch angle as they move toward the minimum field near the equator and then defocusing the pitch angle distribution as they move toward the conjugate ionosphere. These processes therefore are competing, pushing the electrons in opposite directions in pitch angle.

Examination of the left-hand side (LHS) of (11) reveals that electrons will not move along simple Cartesian grid lines. Figure 3 shows typical paths electrons would travel in the $s-\mu$ plane. They are also drifting up and down in energy, so Figure 3 is not a level cutaway at a given energy. This drifting as the particles move along the field line means that the timescale for the distribution function to change in energy and pitch angle is less than a plasmaspheric bounce period, $\tau_{B}$ (the time it takes to traverse the field line, mirror in velocity due to the inhomogeneous magnetic field, and traverse back to the starting point). Imposing a Cartesian grid throughout the $(s, E, \mu)$ phase space would require a very small time step and a highresolution numerical technique to decrease undesirable computational effects associated with approximation errors in the $\partial / \partial E$ and $\partial / \partial \mu$ derivatives, making the computation prohibitively cumbersome. It is desirable, then, to pick a new set of variables that would eliminate two of the three drift terms 


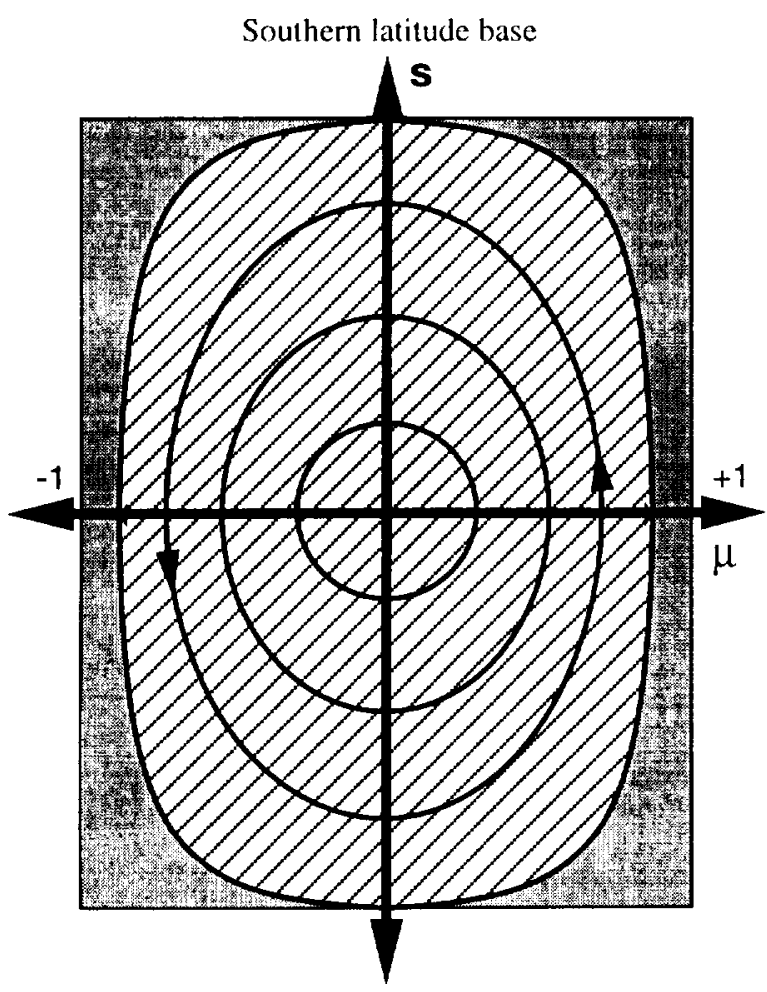

Northern latitude base

Figure 3. Schematic of superthermal electron trajectories in space and pitch angle from the LHS of (11), following Khazanov et al. [1992]. The shaded region is the loss cone, in which particles reach the ionospheres, and the striped region is the trapped zone, in which particles mirror before the base of the flux tube.

on the LHS of (11), thus having velocity-drift timescales much longer than $\tau_{B}$.

To reduce the LHS of (11) to spatial advection only, a transformation of variables must occur, as was performed in the previous descriptions of this model (see, for example, Khazanov et al. [1993]). The new variables should be chosen to eliminate the $\partial / \partial E$ and $\partial / \partial \mu$ derivatives on the LHS of (11) so that $\phi$ becomes a slowly varying function with $s$. Collisions will then be the only process causing $\phi$ to deviate from simple advection along the field line. In general, plasmaspheric collisional processes occur on a timescale longer than $\tau_{B}$. The transformation presented here includes changing the energy and pitch angle variables due to the presence of $B$ and a nonzero $F$.

We will be transforming from $(s, E, \mu)$ to $\left(s, \varepsilon, \mu_{o}\right)$. The new variable set is determined from the characteristics of the LHS of (11) by equating the spatial and energy derivative terms,

$$
\frac{d s}{\mu}=\frac{E \cdot d E}{-e E_{||} E \mu}
$$

and the spatial and pitch angle derivative terms,

$$
\frac{d s}{\mu}=\frac{d \mu}{-\frac{1-\mu^{2}}{2}\left(\frac{e E_{11}}{E}+\frac{1}{B} \frac{\partial B}{\partial s}\right)}
$$

Using an electrostatic definition of the electric field, $E_{11}=$ - $d \Phi / d s$, and introducing an electric potential difference, $\Delta \Phi(s)=\Phi(s)-\Phi\left(s_{r e f}\right)$, integration of (12) yields the new variable $\mathcal{E}$,

$$
\varepsilon(s, E)=E-e \Delta \Phi(s)
$$

which is the total energy of the particle. Substituting this into (13) and integrating reveals the first adiabatic invariant,

$$
\mu_{o}(s, E, \mu)=\sqrt{1-\frac{B_{o} E}{B(s)\left[E-e\left(\Delta \Phi(s)-\Delta \Phi_{o}\right)\right]}\left(1-\mu^{2}\right)}
$$

where the subscript " $o$ " indicates the parameter value at reference point $s_{o}$. The potential differences are measured from an arbitrary reference point, $s_{r e f}$, which does not have to be equal to $s_{o}$.

Using (14) and (15), the kinetic equation (11) can be rewritten in the form

$$
\frac{\beta}{\sqrt{E}} \frac{\partial \phi^{\prime}}{\partial t}+E \mu \frac{\partial}{\partial s}\left(\frac{\phi^{\prime}}{E}\right)=Q^{\prime}+\left\langle S^{\prime}\right\rangle
$$

where $\phi^{\prime}=\phi\left(t, E, \mu, s \rightarrow t, \varepsilon, \mu_{O}, s\right)$ is the differential flux of electrons in the new variable set, $Q^{\prime}$ and $\left\langle S^{\prime}\right\rangle$ have also been transformed, and $E$ and $\mu$ now have the form

$$
\begin{gathered}
E(s, \mathcal{E})=\mathcal{E}+e \Delta \Phi(s) \\
\mu\left(s, \mathcal{E}, \mu_{o}\right)=\sqrt{1-\frac{B(s)\left[\mathcal{E}+e \Delta \Phi_{0}\right]}{B_{o}[\mathcal{E}+e \Delta \Phi(s)]}\left(1-\mu_{0}^{2}\right)}
\end{gathered}
$$

As seen in (16), the LHS of the kinetic equation is now reduced to only advection through the flux tube.

For this study, the collision terms of Khazanov and Liemohn [1995] will be used. While the inelastic collisions with neutral particles only require a shift in energy according to (14), the Coulomb collision operators and the elastic scattering with neutrals involve energy and pitch angle transformations of the form

$$
\begin{array}{r}
\frac{\partial}{\partial E}\left(\frac{\phi}{E}\right)=\frac{\partial}{\partial \varepsilon}\left(\frac{\phi}{E}\right)+\frac{1}{2 E} \frac{1-\mu_{0}^{2}}{\left(\varepsilon+e \Delta \Phi_{0}\right) \mu_{0}} \frac{\partial \phi}{\partial \mu_{0}} \\
\frac{\partial}{\partial \mu}\left[\left(1-\mu^{2}\right) \frac{\partial \phi}{\partial \mu}\right]= \\
+\frac{\left(1-\mu_{0}^{2}\right)\left(\eta-1+\mu_{0}^{2}\right)}{\mu_{0}^{2}} \frac{\partial^{2} \phi}{\partial \mu_{0}^{2}} \\
+\frac{1+\mu_{0}^{2}-2 \mu_{0}^{4}-\eta\left(1-\mu_{0}^{2}\right)}{\mu_{0}^{3}} \frac{\partial \phi}{\partial \mu_{0}}
\end{array}
$$

where

$$
\eta(s)=\frac{\varepsilon+e \Delta \Phi(s)}{\varepsilon+e \Delta \Phi_{o}} \cdot \frac{B_{o}}{B(s)}
$$

and $E$ on the right-hand side is defined above. As before, terms of order $m_{e} / m_{i}$ and the second derivative with respect to energy will be omitted from the kinetic equation calculations [Khazanov et al., 1994], and we come to a structure similar to that described by Khazanov and Liemohn [1995].

\section{Numerical Implementation}

It is now possible to define a Cartesian grid throughout ( $s$, $\mathcal{E}, \mu_{o}$ ) phase space and perform the calculation. A typical tra- 


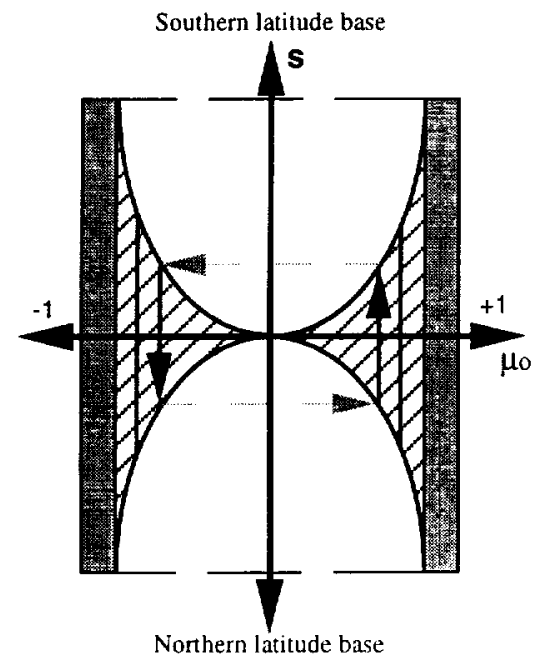

(a)

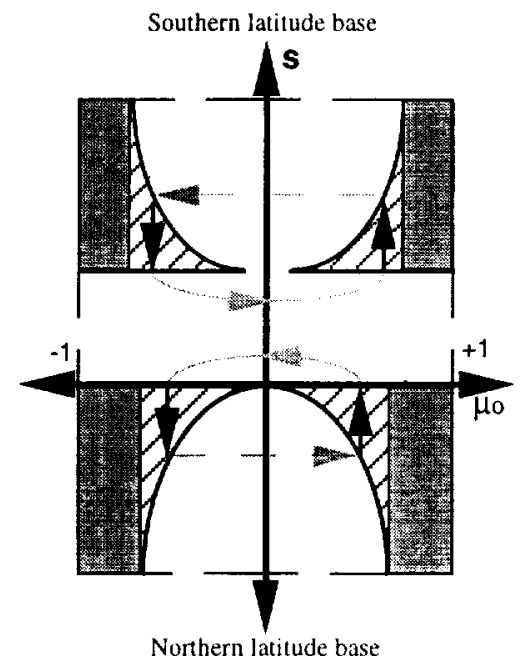

(b)

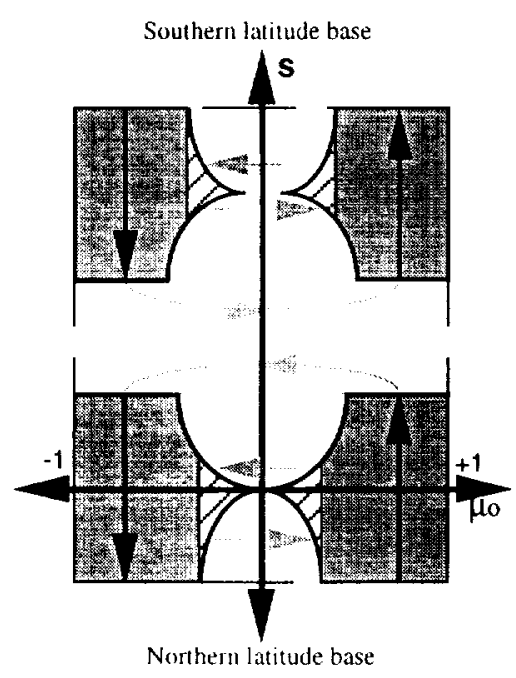

(c)

Figure 4. Schematics of superthermal electron trajectories at constant $\varepsilon$ from the LHS of (16). Shaded and striped regions have the same meaning as in Figure 3, and white indicates forbidden areas outside of the region of existence, where no calculation is necessary.

jectory in the $s-\mu_{o}$ plane at constant $\mathcal{E}$ is shown in Figure $4 \mathrm{a}$, where the shaded region represents pitch angles that penetrate to the ionospheres and the striped region represents the trapped zone in which particles mirror and remain in the plasmasphere. White shows places outside the region of existence, where no calculation will take place. This schematic is found from (15), where $\varepsilon$ has been chosen larger than $\Delta \Phi_{0}+E_{m i n}$. The loss cones (shaded regions) are defined by letting $s=s_{\text {base }}$ and $\mu=0$, and is constant for a given $\varepsilon$. The edge of the trapped zone defining the region of existence is found by setting $\mu=0$ for each altitude along the field line. In the absence of collisions, the particles travel in straight lines in this plane until they reach the edge of the region of existence, whereupon they cross over to the stream flowing in the opposite direction. This crossover point is the mirror point for that particular $\mu_{o}$, where $\mu\left(\mu_{o}, s\right)=0$. The picture is symmetric about the $s$ axis, but not necessarily symmetric about the $\mu_{o}$ axis (this depends on the spatial symmetry of $B$ and $F$ ). The altitude of the $\mu_{o}$ axis is located at the point of minimum $\eta$, defined in (17). As the electrons move from the ionospheres toward the equatorial plane, their kinetic energy changes with $\Delta \Phi(s)$ according to (14), even though they remain on the same $\varepsilon$ level.

We are particularly interested in simulating the superthermal electrons at low energies, since these particles have the largest interaction cross sections with the thermal plasma, so it is necessary to choose an $\boldsymbol{E}$ grid extending down to the intersection of the thermal and superthermal electron populations. However, in the case of plasmaspheric refilling being studied here, $\Delta \Phi(s)$ decreases with increasing altitude up to the equatorial plane (Figure 2). When $|e \Delta \Phi(s)|>\varepsilon-E_{\text {min }}$, the kinetic energy of these particles becomes less than the cutoff $E_{\min }$ at some point $s$ along the field line, because $E=\varepsilon+\mathrm{e} \Delta \Phi(s)$. When this occurs, the region of existence will end at this altitude for this $\varepsilon$, and the $s-\mu_{o}$ plane at constant $\varepsilon$ will look like either Figure $4 \mathrm{~b}$ or $4 \mathrm{c}$. In these cases, the reference altitude for $\mu_{o}$ has shifted down the field line to the point where $\eta(s)$ reaches a minimum and $E$ is still greater than or equal to $E_{\min }$. In
Figure $4 \mathrm{~b}$, this point is the altitude when $E=E_{\min }$, while in Figure $4 \mathrm{c}$ this point occurs lower down the field line, creating a trapped population with both mirror points on the same side of the equatorial plane. As with Figure $4 a$, Figures $4 b$ and $4 c$ are symmetric about the $s$ axis but not about the $\mu_{o}$ axis. In fact, only the $s_{o}$ point will have $\mu_{o}$ defined completely across from -1 to +1 ; for all other altitudes, the region of existence will end before $\mu_{o}$ reaches zero.

Figures $4 b$ and $4 c$ represent decoupled hemispheres for superthermal electron transport. The particles formed in the ionosphere with at an energy that has one of these $s-\mu_{o}$ planes will not reach the conjugate ionosphere; it will be reflected when the potential barrier has removed its field-aligned energy and start to move back toward its source ionosphere. This is shown in Figures $4 \mathrm{~b}$ and $4 \mathrm{c}$ by the curved gray arrows connecting the upward flowing stream with the downward flowing stream at this reflection point. For the purposes of this study, the fluxes of particles are simply mapped to the downward stream. In Figure $4 \mathrm{c}$ the trapped zone does not necessarily reach the reflection altitude. This indicates that particles can become trapped in one hemisphere, mirroring before the equatorial plane, and usually not much above the ionosphere. This trapping mechanism is analogous to the trapped population mentioned in the classification scheme of Lemaire and Scherer [1972]. That paper discussed the various populations present on a polar cap field line, where the ambipolar electric field is important in determining ion outflows. Competition between the magnetic field divergence and the electric potential barrier can sometimes create a trapped electron population with two mirror points along an open field line, as seen in the regions of existence presented in this study. Chiu and Schulz [1978] also discussed this population in the context of parallel electric fields along auroral field lines.

The other aspects of the numerical implementation, initial conditions, and boundary values of each model are the same as in previous works [Khazanov and Liemohn, 1995; Guiter et al., 1995]. However, the ion heat conduction terms in the thermal plasma model are now treated using a fully implicit 
scheme in order to minimize numerical oscillations which occur at shocks when using a Crank-Nicholson scheme. To couple the two models, a driver program is used to alternately call each model, advancing each solution the same time increment and trading the relevant information between the codes. The thermal plasma model is started first, advancing half of a time increment, followed by a full time increment of the superthermal electron model, so the two models leapfrog in time. Using this method, the nonlinearity of the equations due to the self-consistent coupling is substituted for the values at the previous time step or half time step. The effects of this substitution are minimized by choosing a small time step.

\section{Results}

The main purpose of this paper is to demonstrate the effects of self-consistently collisionally and electrodynamically coupling the thermal and superthermal plasmas in a timedependent calculation. This will be shown through plasmaspheric refilling after a geomagnetic disturbance along a flux tube at $L=4$ (geomagnetic equatorial plane crossing at $4 R_{E}$ from the center of the Earth). The simulations start on the dayside (0920 LT, $1400 \mathrm{UT}$ ) on March 21, 1982. An $L=4$ flux tube is a closed field line, and so the simulation range extends from the base of the northern ionosphere through the plasmasphere to the base of the conjugate ionosphere in the southern hemisphere. The conjugate ionospheres are not illuminated symmetrically due to the tilt of the dipole, but the source terms are within $10 \%$ of each other. The initial conditions for the thermal plasma along the field line are shown in Figure 1 of Guiter et al. [1995], and the calculations begin with no superthermal electron population along the field line. These choices are somewhat arbitrary because of a lack of experimental theoretical knowledge of depleted flux tube characteristics, but the use of $n_{e}=0.4 \mathrm{~cm}^{-3}$ at the equatorial plane is reasonable [cf. Singh and Horwitz, 1992].

\subsection{Effects of Electrodynamic Coupling}

To demonstrate some of the effects of the electrodynamic coupling processes included in this study, a comparison between two cases will be shown, with and without the derivations described above. The results marked "With EDC" include the superthermal electron population in the thermal plasma equations (section 2), and include the electric field in the superthermal electron kinetic equation (section 3). The results marked "No EDC" include only the heating rate in the electron energy equation but omit superthermal electrons in the other thermal plasma equations, and do not include the electric field in (11). All other aspects of the simulations are the same.

The influence on the protons is demonstrated in Figure 5, showing the $\mathrm{H}^{+}$bulk velocity from the southern hemisphere stream along the field line after (a) $1 \mathrm{~min}$, (b) $10 \mathrm{~min}$, and (c) $15 \mathrm{~min}$ of refilling. In these plots (as in later figures), distance is counted from the base of the northern ionosphere, so the northern ionosphere is to the left and the southern ionosphere is to the right, with the equatorial plane located at $s=4.51 R_{E}$. Note that a positive velocity indicates flow from north to south, so the large negative velocities mean the protons are flowing toward the northern ionosphere. The new formulation appears to very slightly accelerate the ions, so that by $15 \mathrm{~min}$ the shock front is $500 \mathrm{~km}$ farther downstream

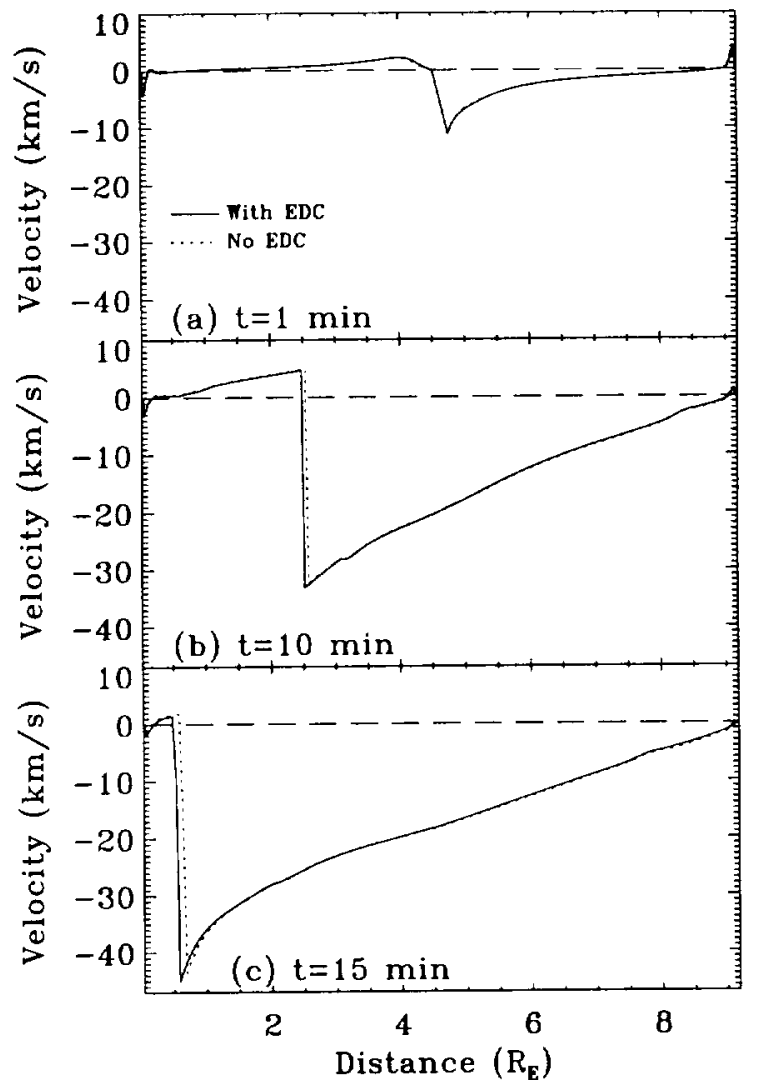

Figure 5. Southern stream $\mathrm{H}^{+}$velocities along the field line with (solid line) and without (dotted line) electrodynamic coupling at (a) $1 \mathrm{~min}$, (b) $10 \mathrm{~min}$, and (c) $15 \mathrm{~min}$ of refilling. The dashed line shows $u=0$. Distances are counted from the base of the northern ionosphere.

than without the new coupling terms. The maximum speed of the shock is also $1 \mathrm{~km} / \mathrm{s}$ higher with the new terms at $15 \mathrm{~min}$. This additional progress of the ions can be explained by the influence of the superthermal electrons on the electric field during the initial stages of the refilling process.

The impact of the superthermal electron terms on the thermal electron population is significant. Figure 6 shows the thermal electron velocity as a function of field-aligned distance at several times, with and without the electrodynamic coupling processes. After $10 \mathrm{~s}$ of refilling time (Figure 6a), the electrodynamic coupling is causing the thermal electrons to stream away from the equator, whereas the thermal electrons stream toward the equator when this process is not included. This is due to the large superthermal electron flux now taken into account in the currentless condition (2). A result such as this could be unstable, but the topic of stability in the thermal and superthermal distributions will be addressed at a later date. After $1 \mathrm{~min}$ (Figure $6 \mathrm{~b}$ ), the results with electrodynamic coupling are beginning to resemble the results without, as the ions flow into the plasmasphere and balance the superthermal electron flux. By 15 min of refilling, as seen in Figure $6 c$, the two results are quite similar, with the only deviations being near the ionospheres. This is because the counterstreaming superthermal electron populations are not balanced at these altitudes, because one stream is near its source and the other stream has traversed the plasmasphere. 


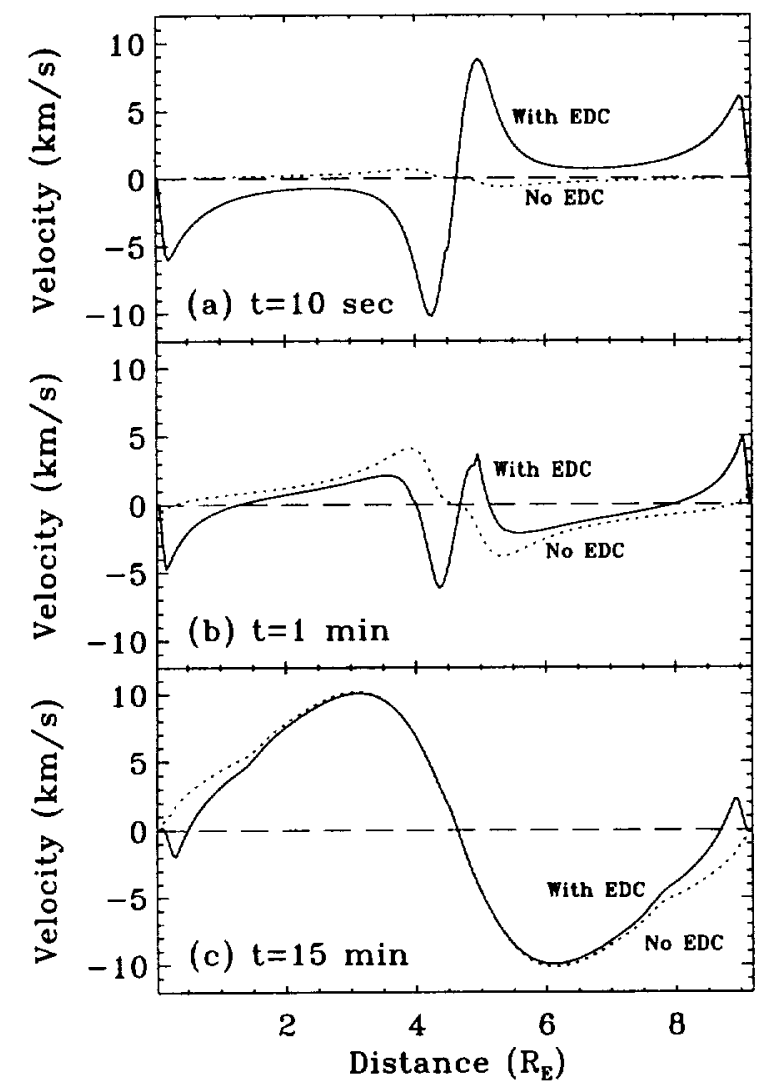

Figure 6. Thermal electron velocities along the field line with (solid lines) and without (dotted lines) electrodynamic coupling at (a) $10 \mathrm{sec}$, (b) $1 \mathrm{~min}$, and (c) $15 \mathrm{~min}$ of refilling. The dashed lines show $u=0$.

From section 3, the use of (16) instead of (11) leads to changes in the superthermal electron results. Figure 7 a shows omnidirectional flux spectra versus kinetic energy after $10 \mathrm{~s}$ of refilling at the topside ionosphere and the equatorial plane, with and without electrodynamic coupling. Omnidirectional flux is defined as

$$
\phi_{\text {omni }}=\frac{1}{2} \int_{-1}^{1} \phi d \mu
$$

The results at $800 \mathrm{~km}$ clearly show the photoelectron production peaks in the $20-30 \mathrm{eV}$ range, and it can be seen that there is a downward shift in the energy of these peaks of around $1 \mathrm{eV}$ in the results with the coupling terms. At 4.51 $R_{E}$, the potential drop is close to $5 \mathrm{~V}$, and the entire distribution has shifted downward accordingly. An increase at low energies due to the coupling is evident in the equatorial results, also due to the downward shift of the distribution function. Coulomb collisions, which have an energy dependence of $E^{-2}$, are more efficient at degrading the superthermal flux levels at lower energies, and so the results with electrodynamic coupling are not a perfectly shifted image of the results without coupling.

Figure $7 \mathrm{~b}$ shows the effects of electrodynamic coupling on the superthermal electron pitch angle distributions. Presented here are equatorial distributions after $30 \mathrm{~min}$ with and without coupling at 5 and $10 \mathrm{eV}$ of kinetic energy. The fluxes that include electrodynamic coupling processes are lower than the results without at small pitch angles, but are higher at larger pitch angles. This effect is due to the decrease in $\mathbf{v}_{\|}$from the field-aligned electric field, causing a shift to lower energies and to larger pitch angles as the electrons move from the ionosphere to the equatorial plane (see Figure 2). The effect is small because the superthermal electron source is the ionosphere. In the plasmasphere, then, the source is in the loss cone, located at small pitch angles, where the $v_{\|}$influence is primarily in energy and not in pitch angle (see equation (11) and Figure 2). Coulomb collisions with the thermal plasma are responsible for scattering electrons into the trapped zone, and the electric field acts to push them further into the trapped zone. For reference, the conventional loss cone ( $B$ influence only) for an $L=4$ flux tube is $5.5^{\circ}$. Here, however, the nonzero $F$ term in (11) expands the loss cone as a function of energy and time. However, even for a case such as $\varepsilon=6 \mathrm{eV}$ and $\Delta \Phi_{0}=-5$ $\mathrm{V}$, the loss cone will only expand to $13.5^{\circ}$.

\subsection{Time Dependence of the Results}

It is interesting to show the development of the solution with respect to time. As mentioned in the Introduction, this is the first model to self-consistently couple the thermal plasma and superthermal electron calculations collisionally and electrodynamically under nonsteady conditions, and here we will demonstrate the time dependence of the results.

With the inclusion of electrodynamic coupling, the total ion density must now equal the thermal and superthermal electron densities combined. In this simulation, the initial

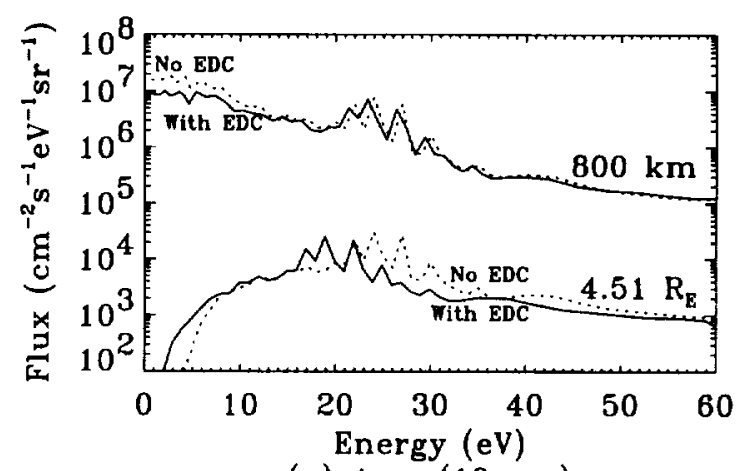

(a) $\phi_{\text {omni }}(10 \mathrm{sec})$

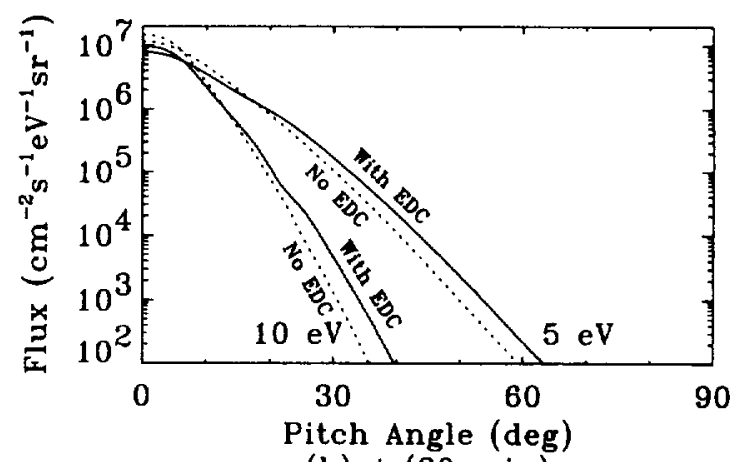

(b) $\phi(30 \mathrm{~min})$

Figure 7. Superthermal electron (a) omnidirectional flux spectra and (b) equatorial pitch angle distributions with (solid lines) and without (dotted lines) electrodynamic coupling. The $\phi_{\text {omni }}$ are shown after $10 \mathrm{~s}$ of refilling at $800 \mathrm{~km}$ (topside ionosphere) and $4.51 R_{E}$ (equatorial plane) along the field line. Pitch angle distributions are shown after $30 \mathrm{~min}$ of refilling at kinetic energies of 5 and $10 \mathrm{eV}$. 


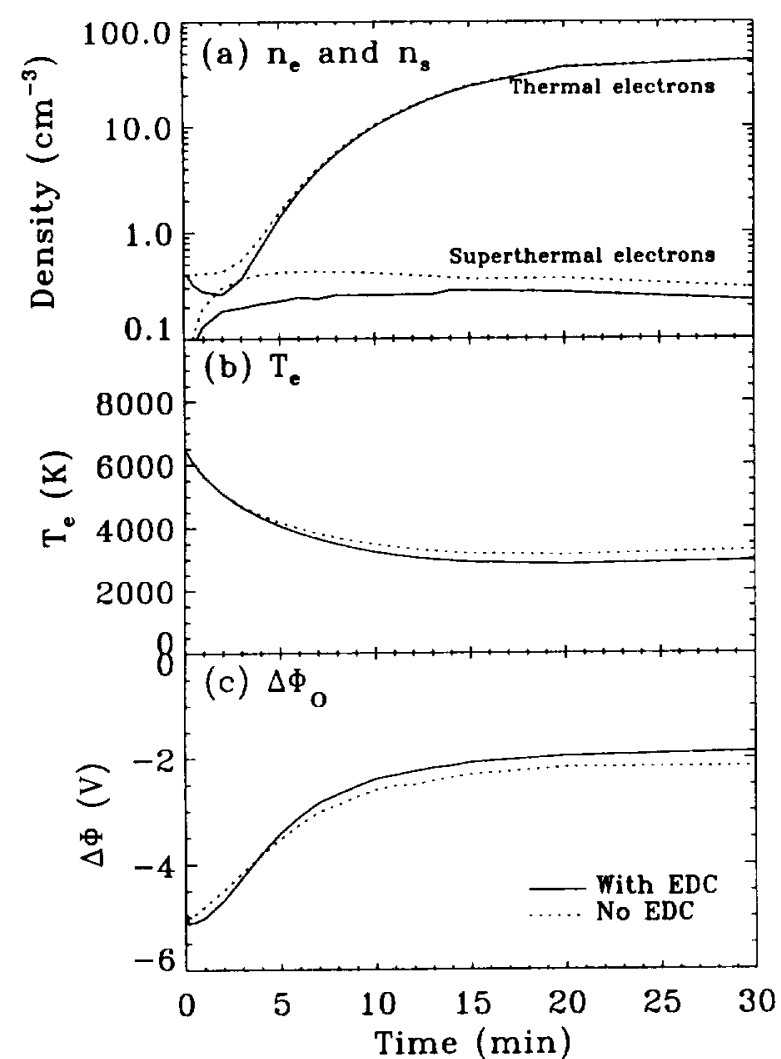

Figure 8. Refilling time dependence of the equatorial values of (a) electron densities, (b) thermal electron temperatures, and (c) electrostatic potential differences with (solid lines) and without (dotted lines) electrodynamic coupling.

thermal electron density at the equatorial plane is $0.4 \mathrm{~cm}^{-3}$. It is conceivable, then, for the superthermal electron density to approach this level and significantly affect the thermal electron density. In Figure $8 \mathrm{a}$, equatorial densities for the thermal and superthermal components of the electron population are shown for the first $30 \mathrm{~min}$ of refilling. For the results with the new coupling terms, the superthermal density has risen to $0.18 \mathrm{~cm}^{-3}$ and the thermal density has dropped to $0.26 \mathrm{~cm}^{-3}$ after $2 \mathrm{~min}$ of refilling. This illustrates the importance of self-consistently including superthermal electrons during events where the thermal plasma is depleted. After 30 min, however, the thermal plasma is 2 orders of magnitude larger than the superthermal density, and the influence of superthermal electrons on the quasi-neutrality condition is greatly diminished. The superthermal densities without coupling are higher than those with coupling; this is because of deceleration due to the electric field. Also notice that the superthermal electron density is dropping slightly after 15 min. This is due to the increased thermal plasma density in the plasmasphere increasing the Coulomb losses of the superthermal electron population.

Figure $8 \mathrm{~b}$ shows the thermal electron temperature at the equatorial plane. The use of the Spitzer conductivity for the thermal heat flux is responsible for a rapid decline in the plasmaspheric temperature. The coupling results have a lower temperature because of the convection shown in Figure 6 transporting additional energy out of the plasmasphere. The temperatures start to increase, very slightly, after $20 \mathrm{~min}$ or so of refilling. This is consistent with the decrease in superthermal electron density and is because of the Coulomb collision heating of the thermal electrons, defined in (8).

In Figure $8 \mathrm{c}, \Delta \Phi$ is shown for the first $30 \mathrm{~min}$ of refilling at the equatorial plane. Here, the reference altitude for the potential is taken at the point of maximum $\Phi$, located in the northern ionosphere near the $F_{2}$ peak. Notice that $\Delta \Phi_{0}$ changes from -5.2 to $-2.2 \mathrm{~V}$ during the first $15 \mathrm{~min}$. The initial $\Delta \Phi_{\mathrm{o}}$ without the superthermal electron influence is $-4.9 \mathrm{~V}$, so the superthermal electrons increase the potential drop by roughly $5 \%$ during the initial stages of the refilling process. The crossover of these two curves is due to the difference in electron temperature shown in Figure $8 \mathrm{~b}$. Initially, the coupling increases the potential drop, but as the thermal electrons cool, the coupling has the opposite effect.

Plate 1 shows the development of the superthermal electron distribution function along the field line by presenting omnidirectional fluxes after $10 \mathrm{~s}, 2 \mathrm{~min}$, and $30 \mathrm{~min}$ of refilling. In Plate 1a, the photoelectron production peaks between 20 and $30 \mathrm{eV}$ are clearly discernible, and they reveal the downward shift of the particles in energy as they move toward the equatorial plane. Plate $1 \mathrm{~b}$ also has the production peaks, but they are beginning to be smoothed away by the increasing superthermal electron flux level around them, by Coulomb losses as the thermal density refills from the ionosphere, and by the decreasing the potential difference. These peaks are barely noticeable after $30 \mathrm{~min}$ in Plate $1 \mathrm{c}$, where the increased Coulomb losses act to smooth the peaks out of the distribution.

Plate la appears to be a butterfly distribution, except this is the $E$-s plane rather than $v_{\|}-v_{\perp}$. There are several reasons for the formation of this distribution. At low energies, the electrons have not yet reached the equatorial region. A $3 \mathrm{eV}$ electron moves at $1000 \mathrm{~km} / \mathrm{s}$, and even if its pitch angle is zero, it will penetrate less than $2 R_{E}$ along the field line into the plasmasphere after $10 \mathrm{~s}$. However, Coulomb collisions are occurring which help to decelerate the faster particles while they are in the plasmasphere, and so the equatorial region is not totally devoid of low-energy superthermal electrons. The production peaks in the $20-30 \mathrm{eV}$ range are formed by the strong He II-30.4 nm solar emission line, which has an energy of $40 \mathrm{eV}$. The ionization potentials of the atmospheric neutral particles create spikes in the electron distribution, and these electrons can escape the ionosphere and travel through the plasmasphere. At higher energies, the streams of superthermal electrons from the two ionospheric connection points of the flux tube have interpenetrated and started flowing down toward the conjugate ionosphere. However, Coulomb collisions are very slow to scatter these electrons into the trapped zone, and the electrodynamic influence is also very weak. Therefore the trapped zone is relatively empty for these energies, and since omnidirectional flux is an average over pitch angle, the flux appears to decrease in the equatorial region. Thus an $E-s$ plane butterfly distribution arises.

Let us now examine the superthermal electron equatorial pitch angle distribution development. Plate 2 shows the time dependence of the 5 and $30 \mathrm{eV}$ kinetic energy electrons. Note that the two plots have different color scales and dynamic ranges. The loss cone fluxes appear to achieve a steady state level within a minute since these pitch angles are connected to the source regions. The trapped zone, however, must have particles scattered into it from the loss cone, and it is clear that this process takes time to complete. It is evident that the $5 \mathrm{eV}$ 


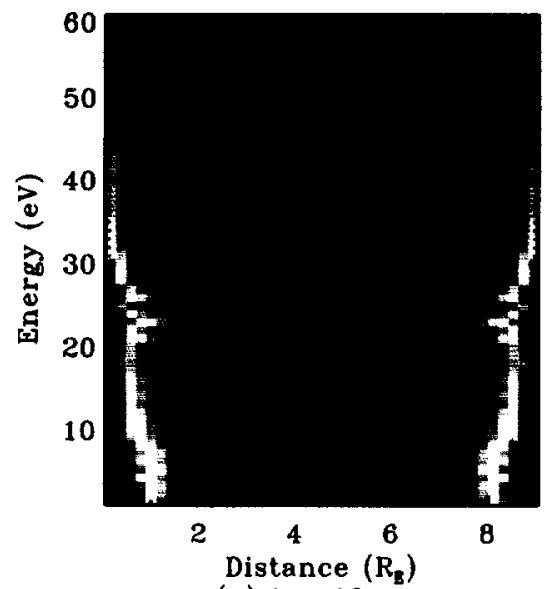

(a) $\mathrm{t}=10 \mathrm{sec}$

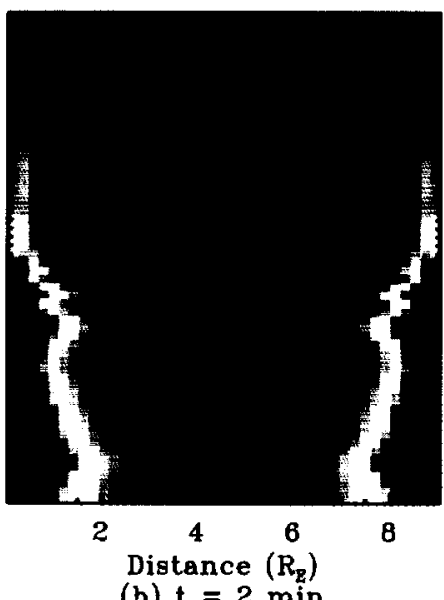

(b) $\mathrm{t}=2 \mathrm{~min}$

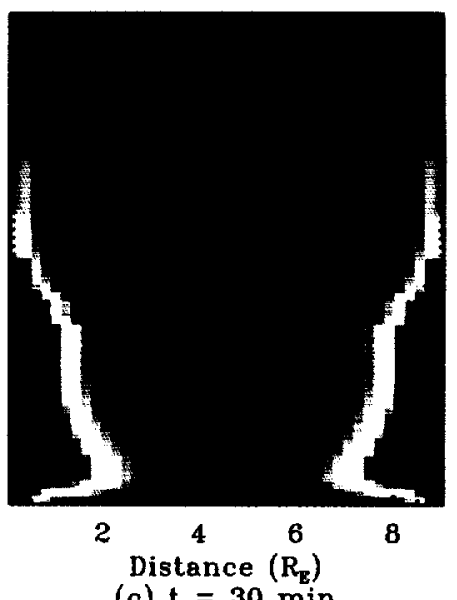

(c) $\mathrm{t}=30 \mathrm{~min}$

Plate 1. Superthermal electron omnidirectional flux spectra along the field line at (a) $10 \mathrm{~s}$, (b) $2 \mathrm{~min}$, and (c) $30 \mathrm{~min}$ of refilling time. Distances are measured from the base of the northern ionosphere. All three plots share the color scale to the right.

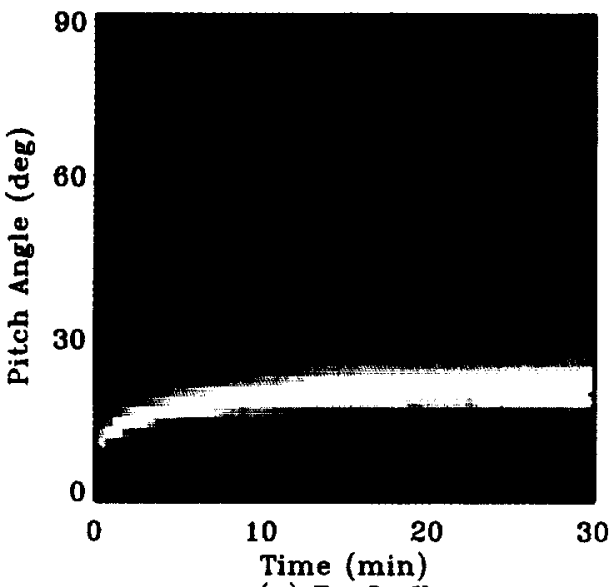

(a) $\mathrm{E}=5 \mathrm{eV}$
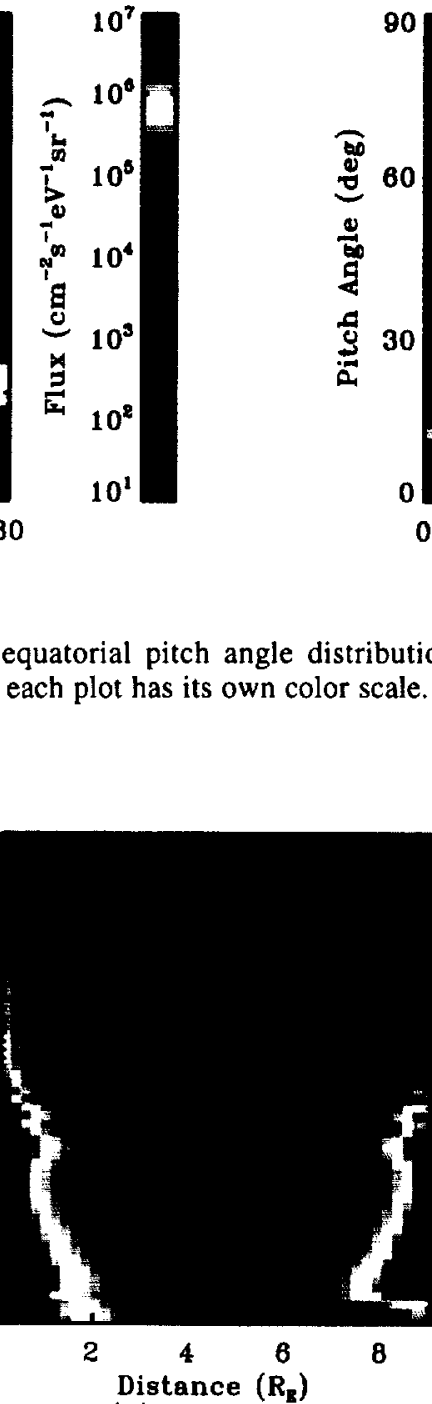

(b) $\mathrm{t}=2 \mathrm{~min}$
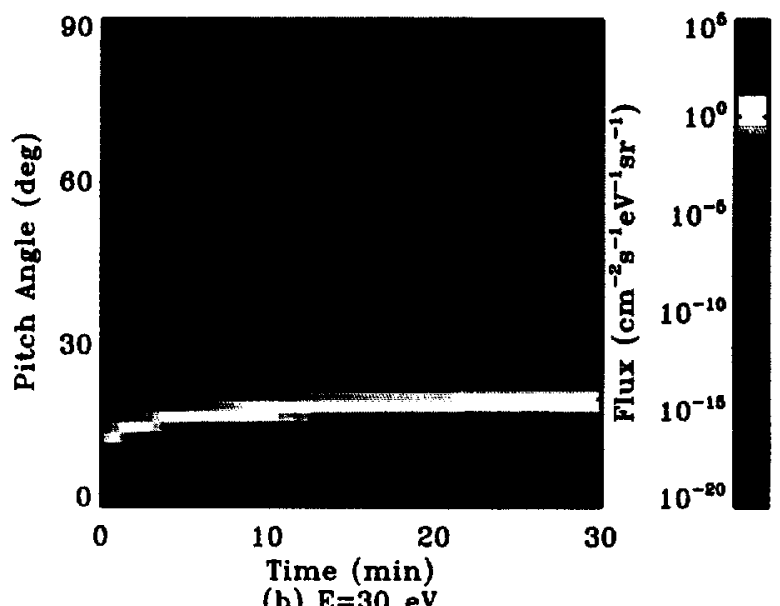

(b) $\mathrm{E}=\mathbf{3 0} \mathrm{eV}$

Plate 2. Superthermal electron equatorial pitch angle distributions vs. refilling time at kinetic energies of (a) $5 \mathrm{eV}$ and (b) $30 \mathrm{eV}$. Note that each plot has its own color scale.

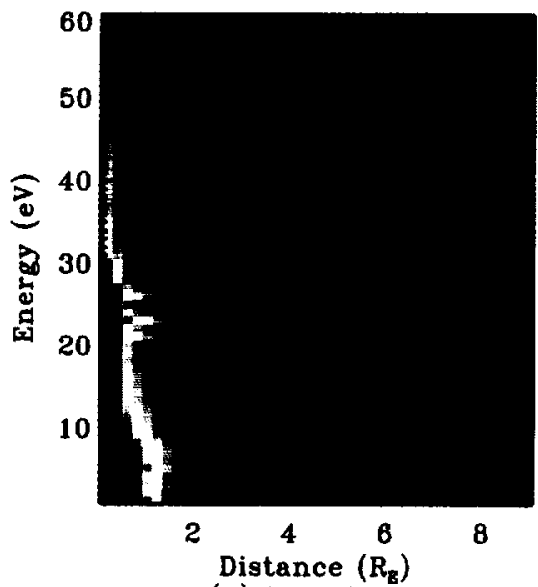

(a) $\mathrm{t}=10 \mathrm{sec}$
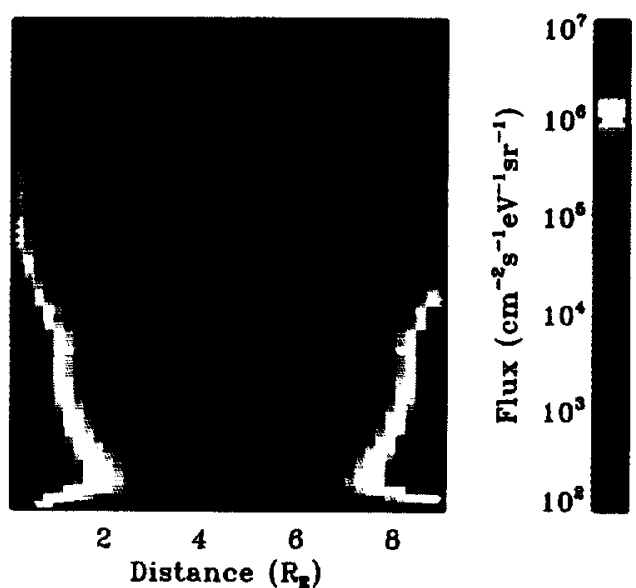

(c) $\mathrm{t}=30 \mathrm{~min}$

Plate 3. Superthermal electron omnidirectional flux spectra along the field line for nonsymmetric illumination at (a) $10 \mathrm{~s}$, (b) $2 \mathrm{~min}$, and (c) $30 \mathrm{~min}$ of refilling time. Distances are measured from the base of the northern ionosphere. Both plots share the color scale to the right. 


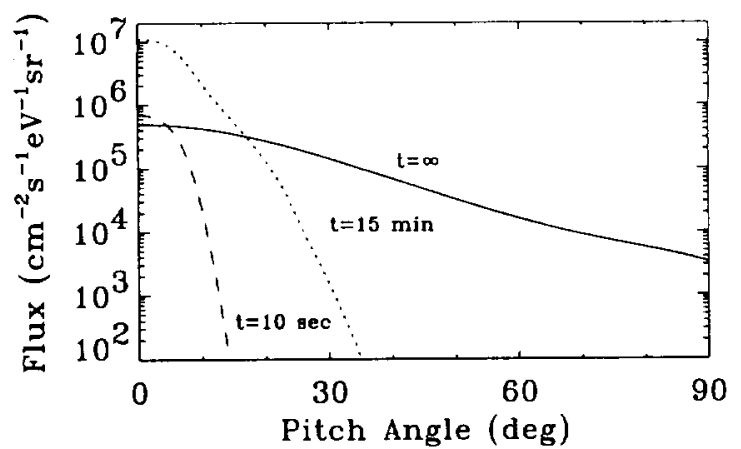

(a) $\phi$ vs. PA

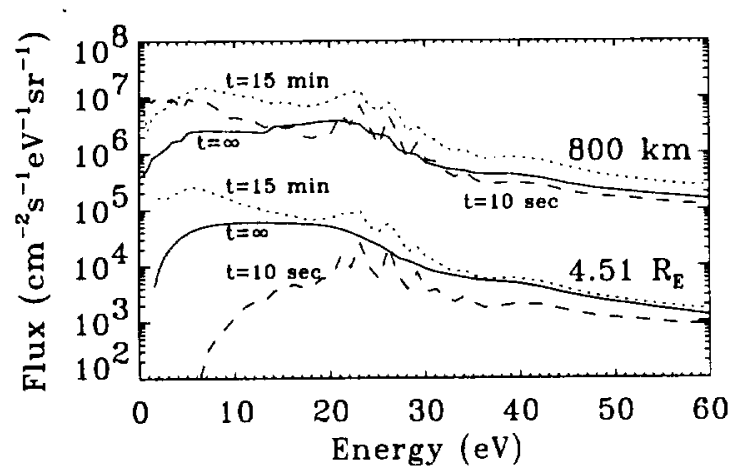

(b) $\phi_{\text {omn } 1}$

Figure 9. Superthermal electron (a) equatorial pitch angle distributions and (b) omnidirectional flux spectra after $10 \mathrm{~s}$ (dashed lines) and $15 \mathrm{~min}$ (dotted lines) of refilling time and steady state (solid lines) results. Pitch angle distributions are at $10 \mathrm{eV}$, and $\phi_{o m n i}$ are at $800 \mathrm{~km}$ (topside ionosphere) and $4.51 R_{E}$ (equatorial plane) up the field line.

fluxes are refilling much faster than the $30 \mathrm{eV}$ fluxes because of the $E^{-2}$ energy dependence of Coulomb collisions. The pitch angles near the loss cone are reaching a steady level, but notice that the distributions deeper in the trapped zone are still refilling after $30 \mathrm{~min}$, and with the thermal plasma still on the rise at this point, electrons will continue to scatter to larger pitch angles for many more hours.

\subsection{Steady State Results}

The process of refilling and the long time period required to reach a steady state level can be accelerated by conducting a simulation that starts with a flux tube filled with thermal plasma. In this case, only the superthermal electron distribution needs to develop, and Khazanov and Liemohn [1995] discussed this jump to steady state for the superthermal electron model. The time step is set to infinity and the model iterates to a converged solution. The two models are still electrodynamically coupled, but the time steps are now iteration steps. The thermal plasma density at the equator is taken to be $2500 \mathrm{~cm}^{-3}$, representing a filled plasmaspheric flux tube. All other aspects of the calculation are the same.

Figure 9a shows $10 \mathrm{eV}$ equatorial pitch angle distributions after $10 \mathrm{~s}$ and $15 \mathrm{~min}$ of refilling and at steady state. Notice that the steady state results have a lower flux at small pitch angles but a higher flux at large pitch angles. This isotropization of the distribution is due to the increased Coulomb scattering cross section in the steady state results. Comparing this steady state distribution with the 5 and $30 \mathrm{eV}$ distri- butions developing in Plate 2, it can be seen that the trapped zone will take a long time to reach this steady-state distribution.

Steady-state omnidirectional fluxes are also compared with the refilling results, shown in Figure 9b. This is similar to Figure $7 \mathrm{a}$, showing spectra at $800 \mathrm{~km}$ and $4.51 R_{E}$ up the field line. Notice that the production peaks are smoothed away in the steady state results by interactions with the thermal plasma. Coulomb collisions have also eroded the low-energy end of the steady state results, and in general decreased the flux levels compared to the results after $15 \mathrm{~min}$ of refilling. The potential difference from ionosphere to equatorial plane is $-1.66 \mathrm{~V}$ for the steady state case, so there is less than a volt difference between this and the potential difference at $15 \mathrm{~min}$.

\subsection{Source Term Effects}

So far, all of the results have been "symmetric," that is, both ionospheric footprints have been illuminated and refilling has occurred from both hemispheres in roughly equal proportions. It is useful to examine the case with nonsymmetric illumination: one sunlit ionosphere and the other in darkness. This will demonstrate the influence of an ionosphere on its conjugate point.

In Plate 3, omnidirectional fluxes along the field line after $10 \mathrm{~s}, 2 \mathrm{~min}$, and $30 \mathrm{~min}$ of refilling are shown without illumination in the southern hemisphere. Compare these results to Plate 1 (with southern illumination). Plate $3 a$ shows a dramatic decrease in the flux of low-energy electrons in the second half of the plasmasphere. However, particles are still traveling to the conjugate hemisphere, and can deposit their energy to the thermal plasma there. Plate $3 b$ is beginning to resemble Plate $1 b$, and Plate $3 c$ is very close to Plate $3 c$. It is still apparent, though, that the fluxes in the southern hemisphere are lower, but it is also clear that plasma from the northern hemisphere flows down to the southern hemisphere to interact with the nonsunlit atmosphere.

Figure 10 shows thermal plasma heating rates along the field line. The refilling results, both with and without southern hemisphere illumination, are shown after $15 \mathrm{~min}$ of refilling. Also shown are heating rates from the steady state

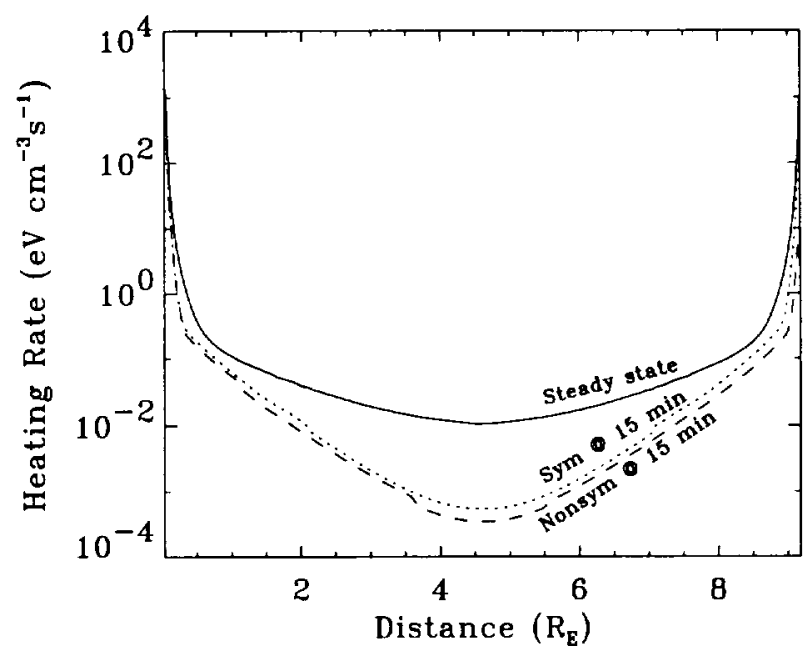

Figure 10. Heating rates along the field line for the steady state case (solid line) and after 15 min of refilling with (dotted line) and without (dashed line) southern hemisphere illumination. 
calculation described in section 5.3. Comparing these results to the heating rates shown by Liemohn and Khazanov [1995] reveals that the steady state calculation is quite similar to the previous steady state results for $L=4$. Notice that the nonsymmetric results are less than the symmetric results for most of the field line, dropping orders of magnitude less in the southern ionosphere. This is consistent with the depleted ionosphere at that end of the flux tube, since after $15 \mathrm{~min}$ the $\mathrm{H}^{+}$stream from the northern hemisphere is just reaching the southern ionosphere.

Heating of the thermal plasma by the superthermal electrons is a slow process during refilling, as can be seen by the factor of 30 difference in the heating rate at the equatorial plane. This is due not only to the increased thermal plasma density but also from the increased superthermal flux in the trapped zone.

\subsection{Data Comparison}

Finally, it is beneficial to show a comparison of results from this model with experimental data. Figure 11 shows omnidirectional fluxes from Atmospheric Explorer E (AE-E) and steady state model results for similar conditions. The data are reproduced from Doering et al. [1976], for day 355 of 1975 at 182 and $365 \mathrm{~km}$ altitude. The solar zenith angles for the two spectra at $50^{\circ}$ and $37^{\circ}$, respectively. Since AE-E flew in a nearly equatorial orbit, the model comparisons are made at $0^{\circ}$ geographic latitude, choosing a local time appropriate with the given solar zenith and assuming the data collection occurred in the morning. Initial profiles for the thermal plasma are taken from the IRI model [Bilitza, 1990] for these conditions in order to start with realistic equatorial iono-

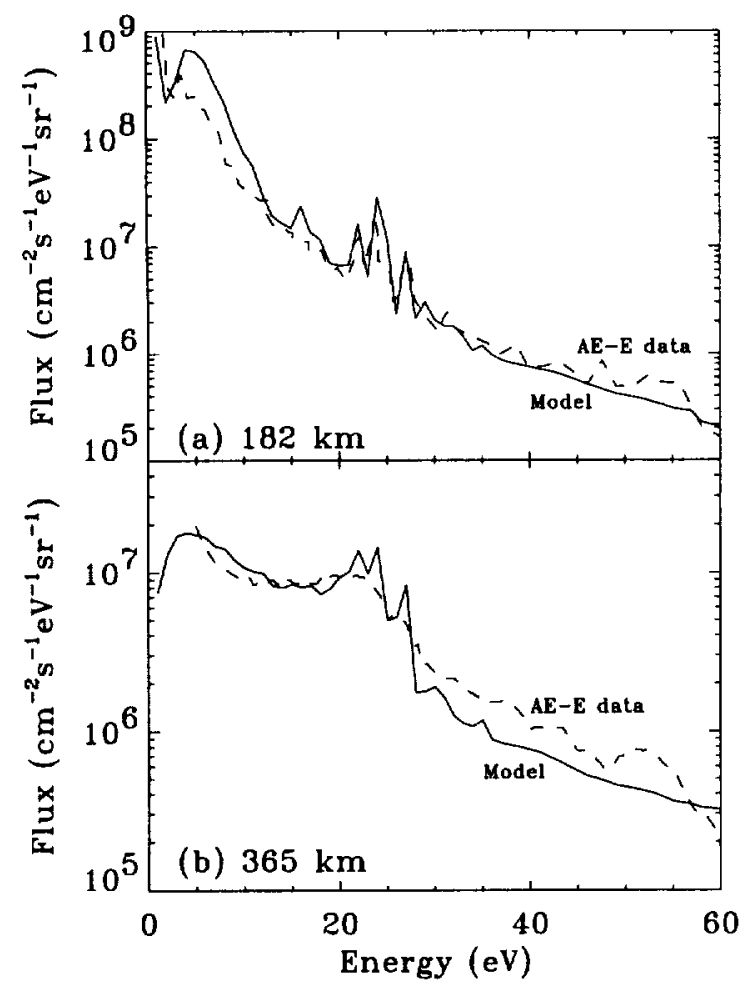

Figure 11. Comparison of model results (solid lines) with AE-E data (dotted lines) at (a) $182 \mathrm{~km}$ and (b) $365 \mathrm{~km}$ altitude on day 355 of 1975 . The satellite data are reproduced from Doering et al. [1976]. spheric densities and temperatures. In Figure $11 \mathrm{a}$, the spectra agree very closely for most of the energy range. The model predicts a slightly higher flux in the $5-15 \mathrm{eV}$ range, but this difference is less than a factor of two. Figure $11 \mathrm{~b}$ also shows good agreement, with the model predicting more definition in the $20-30 \mathrm{eV}$ range and lower fluxes above $30 \mathrm{eV}$ by a factor of less than two. These differences could be explained by uncertainties in the experimental data, differences in the neutral atmosphere or ionospheric plasma profiles, or uncertainties in the collisional cross sections used in the model. The larger fluxes at low energy and the increased definition of the production peaks in the model results indicates that the thermal plasma density from IRI is lower than the actual densities; a higher plasma density would act to smooth out these features of the distribution function. The comparison does show, however, that the model accurately calculates the main features of the photoelectron spectrum.

\section{Discussion}

In this paper, we have discussed the need for simultaneous model calculations of the thermal plasma and superthermal electrons through self-consistently coupling both collisional and electrodynamic processes between the populations. This was achieved by introducing the total electron population (thermal plus superthermal) into the quasineutrality condition, currentless assumption, electron momentum equation, and electron energy equation. Knowing the superthermal electron distribution from numerically solving the field-aligned, guiding center kinetic equation, the former equations are used to find the thermal electron density, bulk velocity, and temperature, as well as the parallel electric field. These quantities are then used in the solution of the kinetic equation to obtain a new superthermal electron distribution. Of special interest is that the ambipolar electric field was included in the kinetic equation, necessitating a new variable transformation to reduce numerical difficulties arising from velocity-space drifts due to the electric and magnetic fields.

Results from calculations based on this new formalism were described in detail. It was shown that the potential drop decreases from -5 to $-2 \mathrm{~V}$ once the initial ion shock fronts penetrate to the conjugate ionosphere. This drop in the potential is due to the increase in thermal plasma density, decrease in thermal plasma temperature, and decrease in superthermal electron flux. From there begins the slow refilling of the plasmasphere, which will take a minimum of several more hours. Removing ion production from one of the ionosphere shows that the sunlit ionosphere can still deposit a significant amount of energy into the dark hemisphere.

In the works of Khazanov et al. [1993] and Liemohn and Khazanov [1995], it was shown that a depleted flux tube could take several hours to reach a steady state level. Here we confirm these results but suggest that superthermal electron refilling will progress faster due to the effects of the fieldaligned potential. These effects include decelerating the electrons as they move towards the equatorial plane and pushing them to larger pitch angles. The pitch angle drift directly contributes to enhanced plasmaspheric trapping, and the energy drift indirectly contributes by moving the particles to a lower energy where Coulomb collisions with the thermal plasma will have a greater scattering cross section and thus the capability of trapping more particles. These trends were pointed out in Figure 7, where the coupled results showed 
enhanced trapping of the superthermal electrons. This effect is small, however, and refilling will still take several hours to complete. The decreased superthermal electron density at the equatorial plane seen in Figure $8 \mathrm{a}$ is due to the decreased loss cone flux from the potential drop. As the potential drop relaxes to smaller values, these loss cone fluxes will return since they are constantly replenished by the ionospheric source.

Influences on the thermal plasma were also calculated. The results indicate that the thermal plasma will refill faster than before due to slightly enhanced proton velocities in the initial shock front. This effect is small because of the large inertia involved in changing the ion distributions. The dramatic influence on the thermal electron velocities shown in Figure 6a has almost disappeared by Figure 6c. This indicates that the thermal plasma will be influenced by the superthermal processes during the early stages of refilling, causing an initial enhancement in the ion population and an initial depletion of the the thermal electron population, but eventually the thermal electron population recovers and refilling will continue as before with the thermal population slightly increased over previous results due to the initial boost. More substantial changes would be expected along auroral or polar cap field lines where the superthermal electron fluxes would not be balanced by particles from the conjugate ionosphere, allowing a longer timeframe for influences on the thermal ions to develop.

As mentioned in the Introduction, we realize a hydrodynamic description of the thermal plasma may not be entirely valid for the low densities involved with the early stages of plasmaspheric refilling. The initial flow of ionospheric plasma through the flux tube will only fill the loss cones of the velocity distribution, resulting in counterstreaming flows. This situation could be unstable, and Schulz and Koons [1972] argued that this two-stream instability is more likely to refill the high pitch angle trapped zone of velocity space than either Coulomb collisions or collisionless shocks. Indeed, they argue against the appearance of a collisionless shock. It would be ideal to have a fully kinetic model of the thermal and superthermal populations, making no distinction between them and modeling the small-angle scattering and possible wave generation and interaction. This model is a step in this direction by including the ambipolar electric field in the kinetic calculation, and we plan to extend the kinetic portion of the model down into the thermal energy regime.

Acknowledgments. This work was supported at the University of Michigan under NASA GSRP grant NGT-51335, at MSFC by the NASA Office of Space Science under UPN 432-20-00, and at the University of Alabama in Huntsville by the NSF grant ATM-9523699. G. V. Khazanov held a National Research Council-Marshall Space Flight Center Senior Research Associateship while this work was performed.

The Editor thanks T. Mukai and M. Schulz for their assistance in evaluating this paper.

\section{References}

Banks, P. M., and G. Kockarts, Aeronomy, Academic, San Diego, Calif., 1973.

Banks, P. M., A. F. Nagy, and W. I. Axford, Dynamical behavior of thermal protons in the mid-latitude ionosphere and magnetosphere, Planet. Space Sci., 19, 1053, 1971.

Bilitza, D., Progress report on IRI status, Adv. Space Res.. IO(11), 3, 1990.

Chiu, Y. T., and M. Schulz, Self-consistent particle and parallel electro- static field distributions in the magnetospheric-ionospheric auroral region, J. Geophys. Res., 83, 629, 1978.

Doering, J. P., W. K. Peterson, C. O. Bostrom, and T. A. Potemra, High resolution daytime photoelectron energy spectra from AE-E, Geophys. Res. Lett., 3, 129, 1976.

Gombosi. T. I., and A. F. Nagy, Time-dependent modeling of fieldaligned current-generated ion transients in the polar wind, $J$. Geophys. Res., 94, 359, 1989.

Gorbachev, O. A., I. M. Sidorov, and G. V. Khazanov, Allowance for thermal flux variations in the model of ionosphere-plasmasphere interactions, Planet. Space Sci., 39, 847, 1991.

Guiter, S. M., and T. I. Gombosi, The role of high-speed plasma slows in plasmaspheric refilling, J. Geophys. Res., 95, 10427, 1990.

Guiter, S. M., T. I. Gombosi, and C. E. Rasmussen, Two-stream modeling of plasmaspheric refilling, J. Geophys. Res., 100, 9519, 1995.

Horwitz, J. L.. Core plasma in the magnetosphere, Rev. Geophys., 25, $579,1987$.

Khazanov, G. V., The Kinetics of the Electron Plasma Component of the Upper Atmosphere (in Russian), Moscow, Nauka, 1979. (English translation, \#80-50707, Nat. Transl. Cent., Washington, D. C., 1980.)

Khazanov, G. V., and M. W. Liemohn, Non-steady-state ionosphereplasmasphere coupling of superthermal electrons, J. Geophys. Res.. $100,9669,1995$.

Khazanov, G. V., M. A. Koen, Y. V. Konikov, and I. M. Sidorov, Simulation of ionosphere-plasmasphere coupling taking into account ion inertia and temperature anisotropy, Planet. Space Sci, 32, 585. 1984.

Khazanov, G. V., T. I. Gombosi, A. F. Nagy, and M. A. Koen, Analysis of the ionosphere-plasmasphere transport of superthermal electrons 1, Transport in the plasmasphere, J. Geophys. Res., 97, 16887, 1992.

Khazanov, G. V., M. W. Liemohn, T. I. Gombosi, and A. F. Nagy, Nonsteady-state transport of superthermal electrons in the plasmasphere Geophys. Res. Lett., 20, 2821, 1993.

Khazanov, G. V., T. Neubert, and G. D. Gefan, Kinetic theory of ionosphere plasmasphere transport of suprathermal electrons, IEEE Trans. Plasma Sci., 22, 187, 1994.

Lemaire, J., and M. Scherer, Ion-exosphere with asymmetric velocity distribution, Phys. Fluids, 15, 760, 1972.

Liemohn, M. W. and G. V. Khazanov, Non-steady-state coupling processes in superthermal electron transport, in Cross-Scale Coupling in Space Plasmas, Geophys. Monogr. Ser., vol. 93, edited by J. L. Horwitz, N. Singh, and J. L. Burch, p. 181, AGU, Washington, D. C., 1995.

Miller, R. H. C. E. Rasmussen, T. I. Gombosi, G. V. Khazanov, and D. Winske, Kinetic simulation of plasma flows in the inner magnetosphere, J. Geophys. Res., 98, 19301, 1993.

Min, Q.-L., D. Lummerzheim, M. H. Rees, and K. Stamnes, Effects of a parallel electric field and the geomagnetic field in the topside ionosphere on auroral and photoelectron energy distributions, $J$. Geophys. Res., 98, 19223, 1993.

Mitchell, H. G., Jr., S. B. Ganguli, and P. J. Palmadesso, Multiple electron species dynamics parallel to a high-latitude field line, Geophys. Res. Lett., 17, 1873, 1990.

Nagy, A. F., and P. M. Banks, Photoelectron fluxes in the ionosphere, $J$. Geophys. Res., 75, 6260, 1970

Nishida, A., Formation of the plasmapause, or magnetospheric knee, by the combined action of magnetospheric convection and plasma escape from the tail, J. Geophys. Res., 7I, 5669, 1966.

Rasmussen, C. E., and R. W. Schunk, Multistream hydrodynamic modeling of interhemispheric plasma flow, J. Geophys. Res., 93, 9831, 1988

Richards, P. G., and D. G. Torr, Thermal coupling of conjugate ionospheres and the tilt of the earth's magnetic field, J. Geophys. Res., 91, 9017, 1986.

Schulz, M., and H. C. Koons, Thermalization of colliding ion streams beyond the plasmapause, J. Geophys. Res., 77, 248, 1972.

Schunk, R. W., and D. S. Watkins, Electron temperature anisotropy in the polar wind, J. Geophys. Res., 86, 91, 1981.

Singh, N., Refilling a plasmaspheric flux tube: Microscopic plasma processes, in Modeling Magnetospheric Plasma. Geophys. Monogr. Ser. vol. 44, edited by T. E. Moore and J. H. Waite Jr., p. 87, AGU, Washington, D. C., 1988.

Singh, N., and J. L. Horwitz, Plasmaspheric refilling, J. Geophys. Res., 97. $1049,1992$.

Singh, N., and W. C. Leung. Numerical simulation of filling a magnetic 
flux tube with cold plasma: Anomalous plasma effects, J. Geophys. Res., 100, 3585, 1995.

Singh, N., H. Thiemann, and R. W. Schunk, Studies on counterstreaming plasma expansion, Phys. Scri., 33, 355, 1986.

Singh, N., G. R. Wilson, and J. L. Horwitz, Comparison of hydrodynamic and semikinetic treatments for a plasma flow along closed field lines, J. Geophys. Res., 99, $11495,1994$.

Tam, S., F. Yasseen, T. Chang, and S. B. Ganguli, Self-consistent kinetic photoelectron effects on the polar wind, Geophys. Res. Lett., 22. 2107, 1995.

Wilson, G. R., J. L. Horwitz, and J. Lin, A semikinetic model for early stage plasmasphere refilling, I, Effects of Coulomb collisions, $J$. Geophys. Res., 97, 1109, 1992.

S. M. Guiter, Center for Space Plasma and Aeronomic Research, University of Alabama in Huntsville, AL 35899.

G. V. Khazanov, M. W. Liemohn, and T. E. Moore, Space Sciences Laboratory, NASA Marshall Space Flight Center, Huntsville, AL 35812.

(Received September 17, 1996; revised December 13, 1996; accepted December 19, 1996.) 